\title{
Towards a model of quintessential inflation
}

\author{
K. Dimopoulos ${ }^{a *}$ \\ ${ }^{a}$ Astroparticle and High Energy Physics group, Instituto de Fisica Corpuscular, \\ Universitat de Valencia/Consejo Superior de Investigaciones Científicas, \\ Edificio Institutos de Paterna, Apartado de Correos 22085, 46071 Valencia, Spain
}

A model of quintessential inflation is presented, which manages to achieve the requirements of both inflation and quintessence with natural values of the mass-scales and parameters.

\section{INTRODUCTION}

Recent observations suggest that the Universe is entering a phase of accelerated expansion [1]. This is also in agreement with other observational facts which favor a Universe dominated at present by a dark energy component [2]. Many consider that this required dark energy is provided by a non-vanishing cosmological constant, which, however, needs to be extremely fine-tuned [3]. The problem may be overcome if the necessary dark energy is provided by a scalar field, called quintessence, whose energy density comes to dominate the Universe today [- Unf. Unfortunately, some fine-tuning is still required for the parameters of the quintessential potential [5]. Moreover, quintessence introduces several other problems, such as tuning of initial conditions.

A possible solution to the above puzzle is linking the quintessence paradigm with inflationary theory. This is a rather natural course of action since both scenarios are based on the same idea, i.e. that the Universe undergoes accelerated expansion when dominated by the potential energy of a scalar field $\phi$, which rolls down its almost flat potential. In such models the quintessence field is identified with the inflaton.

Quintessential inflation has many advantages. For a start, the introduction of yet another scalar field, whose nature and origin is unaccounted for, is avoided. Furthermore, a single theoretical framework can be used to accommodate both

\footnotetext{
${ }^{*}$ This work is done in collaboration with J.W.F. Valle and has been supported by DGICYT grant and the EU under the contract ERBFMRX-CT96-0090.
}

processes. Also, it is possible to avoid introducing and tuning too many mass scales and model parameters. In that way one may solve, or at least link, the fine-tuning problems of quintessence with that of inflation. Finally, one dispenses with several other problems such as the fine tuning of initial conditions, which for the quintessence part are determined by the end of inflation, whereas for inflation itself they can be simply chaotic.

Certain special requirements need to be satisfied in order to construct a successful quintessential inflationary model. Firstly, $V(\phi)$ should feature a "quintessential tail", $V(\phi \rightarrow \infty) \rightarrow 0$, so that $\phi$ has not reached the minimum yet. Secondly, $\phi$ should not be coupled to the Standard Model (SM) fields in order not to decay into SM particles at the end of inflation but, instead, survive until today. Such a "sterile" inflaton dispenses also with one typical fine tuning problem of inflation, mainly that the couplings of the inflaton to the SM should be large enough to ensure prompt reheating but also small enough not to destabilize the flatness of the slow-roll potential. In quintessential inflation reheating is achieved only through gravitational particle production.

Few models of quintessential inflation exist in the literature [6] [7], because it is rather difficult to manage in a single stroke to solve the horizon, flatness, monopole and coincidence problems, while also providing the seeds for Large Scale Structure (LSS) formation and for the anisotropies of the Cosmic Microwave Background Radiation (CMBR). Our model manages to achieve all the above while using natural values for its mass-scales and parameters. 


\section{THE MODEL}

Consider the potential [8],

$V(\phi)=M^{4}(\phi)\left[1+\cos \left(\frac{\pi \phi}{\sqrt{\phi^{2}+\mu^{2}}}\right)\right]$

and

$M(\phi) \equiv M\left\{\frac{M}{\mu}+\frac{\mu}{M} \frac{\sqrt{z} \exp \left(-z \phi / m_{P}\right)}{\cosh (\lambda \phi / \mu) \cos [\omega]}\right\}$

where $[\omega] \equiv\left(\frac{\omega \pi \phi}{\sqrt{\phi^{2}+\mu^{2}}}\right), 0<z, \lambda, \omega \lesssim 1, M \ll \mu$ and $m_{P} \equiv M_{P} / \sqrt{8 \pi}$ with $M_{P}$ being the Planck mass. In order for $V(\phi \rightarrow \infty) \rightarrow 0$, we require $\omega<\frac{1}{2}$. From the above we have,

$$
\begin{aligned}
& V^{\prime}=-(V / \mu) \times \\
& \left\{\frac{4\left[z_{m}+\lambda \tanh (\lambda \phi / \mu)\right]-4 \omega \pi \tan [\omega]\left[1+(\phi / \mu)^{2}\right]^{-3 / 2}}{\frac{1}{\sqrt{z}}\left(\frac{M}{\mu}\right) \cosh (\lambda \phi / \mu) \exp \left(z \phi / m_{P}\right) \cos [\omega]+1}+\right. \\
& \left.\quad+\pi \tan \left(\frac{\pi \phi / 2}{\sqrt{\phi^{2}+\mu^{2}}}\right)\left[1+(\phi / \mu)^{2}\right]^{-3 / 2}\right\}
\end{aligned}
$$

where the prime denotes derivative with respect to $\phi$ and $z_{m} \equiv z\left(\frac{\mu}{m_{P}}\right)$.

The equations of motion are,

$\ddot{\phi}+3 H \dot{\phi}+V^{\prime}=0$

$H^{2}=\frac{\rho}{3 m_{P}^{2}}$

$d\left(a^{3} \rho\right)=-p d\left(a^{3}\right)$

where $H \equiv \dot{a} / a$ is the Hubble parameter with $a(t)$ being the scale factor of the Universe and the dot denotes derivative with respect to the cosmic time t. Also, $\rho$ and $p$ are the total energy and pressure density of the Universe respectively for which,

$\rho=\rho_{\phi}+\rho_{B} \quad p=p_{\phi}+p_{B}$

where the subscript $B$ denotes the background energy and pressure density which satisfy the equation of state, $p_{B}=w_{B} \rho_{B}$, where $w_{B}=1 / 3\{0\}$ for the radiation $\{$ matter $\}$ era. Similarly,

$w_{\phi} \equiv \frac{p_{\phi}}{\rho_{\phi}}=\frac{\dot{\phi}^{2}-2 V(\phi)}{\dot{\phi}^{2}+2 V(\phi)}$

The Universe is undergoing accelerated expansion when $\rho_{\phi}>\rho_{B}$ and $w_{\phi}<0$.

\section{INFLATION}

During inflation (4) and (5) become,

$$
3 H \dot{\phi}+V^{\prime}=0 \quad H^{2} \simeq V / 3 m_{P}^{2}
$$

The slow roll conditions are $\varepsilon, \eta<1$ where,

$\varepsilon \equiv \frac{m_{P}}{\sqrt{6}} \frac{\left|V^{\prime}\right|}{V} \quad \eta \equiv \frac{m_{P}^{2}}{3} \frac{\left|V^{\prime \prime}\right|}{V}$

When $\phi=\phi_{\varepsilon}$ such that $\varepsilon\left(\phi_{\varepsilon}\right)=1$ inflation is terminated. For $\lambda \sim 1$ we have, $\phi_{\varepsilon} \approx 0.1 \mu$ Thus, during inflation $\phi \ll \mu$ and,

$V \simeq 2 z^{2} \mu^{4}=$ const. $\quad V^{\prime} \simeq-8 z^{3} \frac{\mu^{4}}{m_{P}}$

Using these we find,

$N=\int_{\phi_{\varepsilon}}^{\phi_{N}} \frac{V}{V^{\prime}} \frac{d \phi}{m_{P}^{2}} \Rightarrow \phi_{N} \simeq \phi_{\varepsilon}-4 z N m_{P}$

where $N \equiv \int_{\varepsilon}^{N}(d a / a)$ is the remaining number of e-foldings until the end of inflation.

\section{INFLATIONARY REQUIREMENTS}

\subsection{Seeding LSS and CMBR}

The amplitude of the density perturbations is,

$\frac{\delta \rho}{\rho} \simeq \frac{H^{2}}{\dot{\phi}} \simeq-\frac{1}{\sqrt{3}} \frac{V^{3 / 2}}{m_{P}^{3} V^{\prime}} \simeq \frac{1}{2 \sqrt{6}}\left(\frac{\mu}{m_{P}}\right)^{2}$

COBE observations constrain the above as,

$\frac{\Delta T}{T} \simeq \frac{\delta \rho}{\rho} \simeq 10^{-5} \Rightarrow \mu \simeq 1.7 \times 10^{16} \mathrm{GeV}$

Thus, $\mu$ is given by the scale of grand unification.

Let us turn to the power spectrum of density perturbations. The spectral index is defined as,

$n_{s} \equiv 1+\frac{d \ln P_{k}}{d \ln k}$

where $k^{-1} \sim H e^{N}$ is the relevant scale at the end of inflation and, $\left(P_{k}\right)^{1 / 2} \sim \frac{\delta \rho}{\rho}(k)$. After some algebra one finds [8],

$n_{s}-1 \simeq 8 \lambda^{2}-2\left(4 \omega^{2}-\frac{1}{2}\right) \pi^{2}$ 
¿From $\mathrm{COBE}, n_{s} \approx 1$, which is ensured by taking,

$2 \sqrt{2} \lambda \approx \pi \sqrt{8 \omega^{2}-1}$

Thus, we require $\omega \geq \frac{1}{\sqrt{8}}$ so that,

$$
\frac{1}{\sqrt{8}} \leq \omega<\frac{1}{2}
$$

\subsection{Horizon \& Flatness}

The Horizon and Flatness problems are solved if the scale $H_{0}^{-1}$ of the horizon at present did exit the horizon during the inflationary period. The remaining e-foldings $N_{H}$ when this happened are,

$N_{H} \simeq \ln \left(\frac{T_{0}}{H_{0}}\right)+\ln \left(\frac{H_{\text {end }}}{T_{\text {reh }}}\right)$

where $T_{\text {reh }}$ is the reheating temperature. Using (12) we have the constraint,

$$
z \leq \frac{\phi_{\varepsilon}}{4 N_{H} m_{P}}
$$

\section{AFTER THE END OF INFLATION}

\subsection{Reheating}

Although the inflaton does not decay into a thermal bath of SM particles, particle production is generated on gravitational grounds. The gravitationally generated fields have an approximately scale invariant spectrum with amplitude given by the Hawking temperature [9] 10]. Consequently,

$T_{\text {reh }} \sim H_{\text {end }} / 2 \pi$

In view of (19) this gives, $N_{H} \simeq 69$. Therefore, (20) suggests that $z \sim 10^{-6}$. Such a small $z$ ensures the validity of the slow-roll conditions. Using (5) we find, $T_{\text {reh }} \sim 10^{9} \mathrm{GeV}$. Thus, grand unification is not restored so that there is no monopole production. Moreover, such a low $T_{\text {reh }}$ satisfies also the gravitino constraints.

\subsection{Kination}

One can write (4) as,

$$
\frac{\dot{\rho}_{\text {kin }}}{\dot{V}}=-1-3 H \frac{\dot{\phi}}{V^{\prime}}
$$

where $\rho_{\text {kin }} \equiv \frac{1}{2} \dot{\phi}^{2}$. This suggests, $\dot{\rho}_{\text {kin }} \gg \dot{V}$ during inflation. Since $\varepsilon=1 \Leftrightarrow \rho_{\text {kin }} \simeq V_{\text {end }}$, after the end of inflation $\rho_{\text {kin }}$ dominates over $V$. Then $\rho_{\phi}=p_{\phi}=\rho_{\text {kin }}$, which, using (6), gives, $\rho_{\phi} \propto a^{-6}$.

Gravitational reheating is not prompt. Indeed, $\rho_{B} \sim T_{\text {reh }}^{4} \sim H_{\text {end }}^{4} \ll V_{\text {end }} \sim \rho_{\text {kin. }}$. Thus, the Universe remains $\phi$-dominated. This period of $\rho_{\text {kin }}$ domination is usually called kination or deflation [6] [10]. Because $\rho_{B} \propto a^{-4}$, radiation will eventually dominate as required by the Hot Big Bang.

Since $\rho \propto t^{-2}$ during kination, $a \propto t^{1 / 3}$. Using, (9) and (21) we find that kination ends at,

$t_{*} \sim t_{\text {end }}\left(\frac{m_{P}^{4}}{V_{\text {end }}}\right)^{3 / 2}$

where $t_{\text {end }} \sim m_{P} / \sqrt{V_{\text {end }}}$ is the time when inflation is terminated. In view of (11) we find $t_{*} \sim 10^{-2} \mathrm{sec}$, i.e. earlier than Nucleosynthesis.

Let us find how much does the scalar field roll during kination. In this regime (身) becomes, $\ddot{\phi}+(\dot{\phi} / t) \simeq 0$. Solving we find,

$\phi_{*} \equiv \phi\left(t_{*}\right) \simeq \phi_{\varepsilon}+\frac{\sqrt{3}}{4 \pi} \ln \left(\frac{m_{P}}{V_{\text {end }}^{1 / 4}}\right) m_{P}$

\subsection{Hot Big Bang}

At the onset of the radiation era $\rho_{\text {kin }}$ decreases rapidly as, $\rho_{\text {kin }} \propto t^{-3}$ since $a \propto t^{1 / 2}$. Thus, in little time $\dot{\phi} \rightarrow 0$ and the field freezes at $\phi_{F}$. Indeed, it can be shown [8] that,

$\phi_{F}=\phi_{*}+2 \dot{\phi}_{*} t_{*} \simeq \phi_{\varepsilon}+\frac{\sqrt{3}}{12 \pi}\left[1+3 \ln \left(\frac{m_{P}}{V_{\mathrm{end}}^{1 / 4}}\right)\right] m_{P}(25)$

which evaluates to, $\phi_{F} \simeq 1.68 m_{P} \gg \mu$.

\section{QUINTESSENCE}

When $\phi \gg \mu$ then the potential becomes,

$V(\phi) \simeq \frac{\pi^{2}}{8} \frac{M^{8}}{\phi^{4}}\left[1+\frac{\sqrt{z}}{\cos (\omega \pi)}\left(\frac{\mu}{M}\right)^{2} e^{-\lambda \phi / \mu}\right]^{4}$

The exponential term in (26) dominates when,

$\phi<\phi_{C} \equiv \frac{\mu}{\lambda}\left\{2 \ln \left(\frac{\mu}{M}\right)-\ln [\cos (\omega \pi)]+\frac{1}{2} \ln z\right\}$

The quintessential part of the evolution of $\phi$ can be explored analytically with the use of (目),

$3 H \dot{\phi} \simeq-V^{\prime}(\phi) \Rightarrow-\int_{\phi_{F}}^{\phi(t)} \frac{d \phi}{V^{\prime}(\phi)}=\frac{1}{4}\left(w_{B}+1\right) t^{2}(28)$ 
where the field's acceleration $\ddot{\phi}$ is ignored because $\phi$ lies at the "quintessential tail" of $V(\phi)$. It is found [8] that either $\phi$ remains frozen at $\phi_{F}$ or it follows attractor solutions of the form $\phi=\phi_{\text {atr }}(t)$, so that $V(\phi)=\min \left\{V\left(\phi_{F}\right), V\left(\phi_{\text {atr }}\right)\right\}$.

\section{QUINTESSENCE REQUIREMENTS}

The coincidence problem translates to,

$V\left(\phi_{0}\right)=\Omega_{\phi} \rho_{0}$

where $\phi_{0} \equiv \phi\left(t_{0}\right)$ is evaluated at the present time $t_{0}$ and $\Omega_{\phi} \simeq 0.65$ is the ratio of $\rho_{\phi}$ over the critical density today [2] $\rho_{0} \simeq 10^{-120} \mathrm{~m}_{P}^{4}$.

Moreover, in order to explain the current accelerated expansion of the Universe we require,

$w_{\phi}=\frac{\varepsilon_{0}^{2}-\Omega_{\phi}^{-1}}{\varepsilon_{0}^{2}+\Omega_{\phi}^{-1}}<0$

where $\varepsilon_{0} \equiv \varepsilon\left(\phi_{0}\right)$ and we used (8) and (10).

It can be shown that, if $\phi$ starts following the attractor solutions it is impossible to meet the above requirements in the available parameter space [8]. Thus, we need $\phi_{0}=\phi_{F}$, which requires,

$V\left(\phi_{0}\right) \leq V\left(\phi_{\text {atr }}\left(t_{0}\right)\right)$

\subsection{Regimes}

$\phi<\phi_{C}$ : Quasi-exponential quintessence

The potential reduces to,

$V(\phi) \simeq \frac{(\pi z)^{2} / 8}{[\cos (\omega \pi)]^{4}} \frac{\mu^{8}}{\phi^{4}} e^{-4 \lambda \phi / \mu}$

Using (14) we find from (29) that, $\lambda \simeq 0.224$. Moreover, $\phi_{F} \leq \phi_{C}$ results in, $M \leq 0.82 \mathrm{TeV}$. These values can be shown [8] to satisfy the constraint (31). Finally, (30) gives, $w_{\phi} \simeq-0.836$.

$\phi>\phi_{C}$ : Inverse power-law quintessence

The potential reduces to,

$V(\phi) \simeq \frac{\pi^{2}}{8} \frac{M^{8}}{\phi^{4}}$

Now (29) gives, $M \simeq 2.92 \mathrm{TeV}$. Using this, (31) gives, $\lambda \geq 0.225$, corresponding to practically all the $\omega$-range (18). These values, satisfy $\phi_{F} \geq \phi_{C}$ as required [8]. Finally, (30) gives, $w_{\phi} \simeq-0.228$.

\section{CONCLUSIONS}

We have studied analytically a model of quintessential inflation and showed that it is able to satisfy all the requirements of inflation and quintessence with natural values of the parameters, i.e. $\quad M \sim \mathrm{TeV}$, which is possible to link with supersymmetry breaking or even large extra dimensions, and $\mu \sim 10^{16} \mathrm{GeV}$, which is the grand unification scale. The rest of the parameters also assume natural values, $\lambda, \omega \lesssim 1$ except $z \sim 10^{-6}$, which corresponds to the typical fine tuning for the flatness of inflationary potentials. Even though the theoretical justification of our model is still an open issue we have shown that it is indeed possible to construct successful quintessential inflationary models without the need of any additional fine-tuning for quintessence. In that sense, our model outshines the cosmological constant alternative.

\section{REFERENCES}

1. S.Perlmutter et al., Nature 391 (1998) 51; ApJ 517 (1999) 565; A.G.Riess et al., ApJ 116 (1998) 1009; B.P.Schmidt et al., ApJ 507 (1998) 46; P.M.Garnavich et al., ApJ 493 (1998) L53

2. R.G.Carlberg et al., ApJ 478 (1997) 462; N.A.Bahcall et al., Science 284 (1999) 1481; M.Tagmark, ApJ 514 (1999) L69

3. J.D.Barrow \& F.J.Tipler, The Anthropic Cosmological Principle, Oxford UP (1986) p. 668

4. B.Ratra \& P.J.E.Peebles, PRD 37 (1988) 3406; R.R.Caldwell et al., PRL 80 (1998) 1582; E.J.Copeland et al., PRD 57 (1998) 4686; P.G.Ferreira \& M. Joyce, PRD 58 (1998) 023503; P.J.Steinhardt et al., PRD 59 (1999) 123504

5. C.Kolda \& D.H.Lyth, PLB 458 (1999) 197

6. B.Spokoiny, PLB 315 (1993) 40

7. P.J.E.Peebles \& A.Vilenkin, PRD 59 (1999) 063505; M.Peloso \& F.Rosati, JHEP 12 (1999) 026; W.H.Kinney \& A.Riotto, APP 10 (1999) 387

8. K.Dimopoulos \& J.W.F.Valle, in preparation

9. L.H.Ford, PRD 35 (1987) 2955

10. M.Joyce \& T.Prokopec, PRD 57 (1998) 6022 